\title{
MACINTYRE Y EL MARXISMO: HISTORIA, COMPROMISO Y RAZONES PARA LA ACCIÓN
}

\author{
P. Blackledge y N. Davidson (comps.), Alasdair MacIntyre's Early \\ Marxist Writings: Essays and Articles 1953-1974, Brill, Leiden, \\ 2008, 443 pp.
}

\author{
RAFAel RAMis BaRCELó \\ Departamento de Derecho \\ Universidad Pompeu Fabra \\ rafael.ramis@upf.edu
}

Alasdair MacIntyre's Early Marxist Writings es una contribución algo problemática, pero muy necesaria para conocer con profundidad la obra de Alasdair MacIntyre y, en concreto, sus ligámenes con el marxismo. La necesidad radica en que hasta el momento no existía ningún estudio que recopilara la importante contribución de MacIntyre al debate interno que las diferentes corrientes marxistas sostuvieron en los años cincuenta y sesenta. La dificultad se encuentra en la selección y en la explicación del alcance de ese marxismo. Por eso me gustaría dividir este comentario en dos partes: una que atienda principalmente las cuestiones referentes al estudio del marxismo de MacIntyre y, la otra, que dé cuenta de los problemas que tiene esta selección en el corpus del filósofo escocés.

Antes de continuar, es conveniente presentar las características formales del libro. Se trata de una selección de escritos que abarca desde 1953 hasta 1974, es decir, veintiún años en los que MacIntyre pensó un reducido grupo de temas, aunque utilizó distintos métodos para abordarlos. La selección intenta compendiar los trabajos más relevantes sobre el marxismo, y está precedida por un interesante prólogo de los compiladores, Paul Blackledge y Neil Davidson.

Blackledge, profesor de teoría política en la Leeds Metropolitan University, es hoy por hoy, uno de los mayores conocedores de los vaivenes del marxismo en Gran Bretaña. Sus últimas publicaciones se han centrado en el estudio histórico de la New Left y en la historiografía del marxismo inglés. Muy recientemente publicó una serie de artículos que estudian el pensamiento marxista de MacIntyre (Blackledge 2005, 2008 y 2009) poniendo especial atención en el contexto político en el que se movía el pensador escocés. Neil Davidson es 
investigador en la Universidad de Strathclyde. Se ha dedicado fundamentalmente a dos temas que parecen inconexos: el nacionalismo escocés y la filosofía de MacIntyre (Davidson 2007).

En las páginas siguientes no me centraré tanto en los escritos de MacIntyre, sino más bien en los problemas conceptuales e ideológicos que se desprenden del escrito preliminar, y en los criterios de selección de los trabajos que se recogen en el volumen.

\section{El marxismo en MacIntyre}

El compromiso de MacIntyre con el marxismo fue, durante los años cincuenta y sesenta, una constante en su pensamiento que quedó reflejada en sus escritos, aunque no fue la mayor de sus preocupaciones, ni mucho menos el eje de su pensamiento. Su marxismo, por lo demás, se incardinó en el seno de una discusión que convocó a la mayoría de los intelectuales de la época.

Es muy distinto el valor de los escritos marxistas de MacIntyre si se contempla desde el análisis de la trayectoria del autor o si el tema en cuestión es el estudio histórico y sistemático de la izquierda británica durante la guerra fría. Creo que los autores de la compilación toman como referencia el segundo tema, y esto orienta decisivamente el prisma de estudio.

El libro muestra cómo en los años cincuenta y sesenta MacIntyre tuvo un doble discurso sobre el marxismo: el teórico y el práctico. El primero estuvo unido siempre a una serie de cuestiones ligadas a la filosofía de la religión y a la filosofía de la historia, dos temas que no parecen interesar excesivamente a los autores. Sin embargo, la principal preocupación de MacIntyre no era ni el cristianismo ni el marxismo ni la filosofía analítica, sino más bien un problema histórico - y hasta escatológico - que percibía como el desastre propio de la época en que le había tocado vivir.

El problema se situaba en el orden vivencial, pero adquiría una dimensión teórica cuando percibía que en ello estaba en juego la gran cuestión filosófica de su época: la falta de razones para la acción. MacIntyre comprendió el "desierto moral" como la consecuencia inevitable de una época en la que la razón teórica y la razón práctica estaban escindidas sin que hubiera manera de juntarlas.

Antes de estudiar con profundidad la llamada "falacia naturalista" en Kant y Hume (MacIntyre 1978), el escocés era ya consciente de que ni en su época, ni en los siglos precedentes inmediatos, había razones teóricas para la acción. Su primer estudio de importancia ligó el marxismo con el cristianismo, para demostrar — primero a sí mismo 
y después a los demás - que con la ayuda de estas dos ideologías que él secundaba se podían aducir algunas razones para actuar. Así, su estudio teórico se centraba en el ámbito de la religión (cristianismo) y de la historia (marxismo), dos ideologías que se unían para dar cuenta del problema escatológico del mundo futuro (MacIntyre 1953).

Sólo en la medida en que su cristianismo protestante - muy apegado a la línea fideísta de Bultmann - y el marxismo - en su vertiente comunista ortodoxa - resultaban cada vez más insuficientes para explicar el desastre de la época, MacIntyre se dio cuenta de que el problema era irresoluble. De hecho, en los estudios sobre "Philosophical Theology" (la filosofía de la religión que se enseñaba en Oxford durante los años cincuenta y sesenta) se preocupó por saber si realmente existía un lenguaje apto para estudiar analíticamente la redención y la salvación y, si en efecto, éstas eran las razones fundamentales que movían o debían mover la historia.

A finales de los años cincuenta, el filósofo escocés se fue alejando de la creencia cristiana y, al mismo tiempo, para paliar su vacío existencial, fue comprometiéndose más con dos de los movimientos sociales e intelectuales de la época: la New Left y, en menor medida, el psicoanálisis. Con respecto al primer caso, MacIntyre se dedicó cuatro o cinco años (aproximadamente, 1958-1963) a pensar seriamente en los problemas de la izquierda. Como el cristianismo ya no podía dotar de sentido a la razón teórica, la reflexión sobre la religión tuvo un peso menor. Quedaba, sin embargo, la cuestión de la historia, que era el eje de su primer libro, a la que dedicó interesantes estudios desde el prisma marxista, como podrá verse más adelante.

Abandonar el cristianismo le propició un interés subyugante por el psicoanálisis, pues tal vez la razón escindida podía estudiarse desde allí, y encontrar entonces algunas soluciones. De hecho, pese a que no existe - por el momento - un trabajo satisfactorio acerca de la preocupación de MacIntyre por el psicoanálisis, ${ }^{l}$ hay que decir que The Unconscious (MacIntyre 1958) sólo puede entenderse como la búsqueda de unas pulsiones que pudieran ser las bases de la acción individual y colectiva, ya que no había, en puridad, "razón" práctica.

Por lo tanto, la New Left se benefició del análisis histórico de MacIntyre y de su participación en los debates morales y políticos. Era la época en la que el filósofo escocés se había quedado sin el referente cristiano en el que había sido educado, y se había volcado en este movimiento crítico con las vacilaciones de los partidos comunistas tanto británico como estadounidense frente al "socialismo con

${ }^{1}$ Algunas referencias a esta cuestión pueden verse en Ramis Barceló 2010. 
rostro humano". Esta razón coyuntural contribuyó a que MacIntyre fuera testigo privilegiado de uno de los momentos más importantes en la configuración de los movimientos de izquierdas en el mundo anglosajón. De ahí que intentara vincular su anticomunismo partidista con los cambios que iban sucediendo (McMylor 1994, p. 12).

Sin embargo, pese a lo que puede desprenderse del libro de Blackledge y Davidson, MacIntyre no era un autor interesado en la práctica política, sino un profesor desorientado, un navegante en un mar turbulento que buscaba una tabla a la que aferrarse, después de haberse roto su frágil embarcación. En el año 1959, el problema seguía siendo la falta de razones para la acción. Que MacIntyre estuviera ligado durante una década al marxismo (1953-1963) y que durante otra (1963-1973) siguiesen vivos algunos temas propios de esa ideología, creo que se debe más a la oportunidad histórica y a la moda "marxista" entre los universitarios, que a un verdadero interés político. De lo que no tengo ninguna duda es de que si resistió más en el marxismo "militante" - en sus diferentes formas - que en el cristianismo protestante o en el estudio del psicoanálisis no fue por la fortaleza de las razones de aquél, sino por todo lo que oponía al capitalismo liberal.

Con esto se llega al segundo de los grandes temas de MacIntyre, que permean toda su obra y, en buena medida, la explican. He aludido antes al problema de la escisión entre la razón teórica y la razón práctica, que es la consecuencia de un desastre en el orden epistemológico y moral, pero no había mencionado la causa. La etiología de los males del hombre contemporáneo se encuentra en el liberalismo, que creó el Estado-nación.

MacIntyre culpó durante toda su juventud (y continúa haciéndolo a sus ochenta años) al capitalismo y al liberalismo de casi todos los males de la sociedad occidental. El empirismo y el criticismo (Locke, Hobbes y Kant) representan una filosofía escindida y fundada sobre cierto nihilismo, pues, de acuerdo con MacIntyre, el Estado-nación se articuló a partir de la destrucción de las fidelidades y de los cuerpos intermedios (Perreau-Saussine 2005, p. 40).

El sujeto autónomo y atomizado encarnaba la falta de valores comunes del Estado-nación. Era un ciudadano nuevo que había sido arrancado de la historia y de su grupo social y cultural para convertirse en un ser perdido por la idolatría del capital. El marxismo tenía un gran atractivo para MacIntyre, porque al fragmentar la sociedad liberal en varias capas sociales, era capaz de dar un discurso a cada una de ellas. De la fricción que éstas tenían entre sí, aparecieron las inevitables tensiones dialécticas que permitieron que cada persona 
- social y culturalmente- se sintiera partícipe de un grupo. Era la sociedad y la cultura de los obreros, forjada en contraposición a la de los capitalistas.

A partir de este contexto cobra sentido el itinerario "marxista" de MacIntyre, muy bien hilvanado por Blackledge y Davidson. El estudio preliminar de estos autores es descriptivo y da cuenta de los tres grandes estadios en la trayectoria "marxista": su breve y violenta incursión en el Partido Comunista, su participación activa en la New Left y, finalmente, a principios de los años setenta, su contribución como miembro heterodoxo de la Internacional Socialista.

Los autores explican muy bien el contexto en el que MacIntyre escribió sus obras. En un primer momento, en 1948, la influencia directa del helenista George Thompson fue decisiva para su escarceo con el Partido Comunista, y su obra Marxism: An Interpretation da fe de sus forcejeos con el comunismo (Blackledge y Davidson 2008, pp. xxi-xxii).

Por defender el cristianismo evangélico y el marxismo de los $M a$ nuscritos de filosofía y de economía, MacIntyre se vio envuelto en el inmenso revuelo intelectual que ocasionó el octubre húngaro de 1956. Los partidos Comunista y Laborista se quedaron cortos para hacer frente a un problema que traspasaba los límites de la izquierda institucionalizada. En el debate de la incipiente New Left (Blackledge 2005), en discusión con Edward Thompson, Harry Hanson o Charles Taylor, MacIntyre sentó tres ideas básicas que resumen muy bien su "pensamiento marxista", tal y como Blackledge y Davidson lo explican: por una parte, la oposición frontal al estalinismo, por otra, la vuelta a Marx y, finalmente, la necesidad de conservar el legado intelectual de Marx — no el de los marxistas - como discurso para enfrentarse al capitalismo liberal.

Su oposición al estalinismo fue radical, y no toleró los coqueteos consecuencialistas de Thompson: para MacIntyre, como manifestó en "Notes from the Moral Wilderness" (Blackledge y Davidson 2008, cap. 5), el estalinismo era una aberración que quería hacer coincidir una realidad brutal con el devenir histórico del marxismo. MacIntyre se opuso al formalismo de Thompson, que presentaba un humanismo más acomodaticio al llamado "socialismo real". Hanson había escrito una carta abierta contra Thompson, alegando una suerte de deontologismo kantiano, a la cual MacIntyre replicó rápidamente. La idea del filósofo escocés — que después desarrolló en "Hume on 'Is' and 'Ought" y otros trabajos (véase MacIntyre 1978) — es que de Kant no se pueden sacar razones para la acción y que no se puede 
kantianizar a Marx. Al contrario, MacIntyre —antes que muchos otros pensadores de su generación - fue un estudioso de Marx, sin ismos.

De hecho, durante su juventud MacIntyre se interesó en Marx, a quien tuvo como bandera para estimular a los diferentes partidos que se proclamaban hijos de este pensador alemán. A medida que la New Left fue institucionalizándose cada vez más, MacIntyre mostró que él no tenía un objetivo político, sino moral y epistemológico, como a veces apuntan de pasada Blackledge y Davidson. Para MacIntyre, Marx encarnaba al autor que no había escindido la razón teórica de la razón práctica, algo que apenas importaba a otros intelectuales de la época.

Por una razón igualmente teórica y como consecuencia de la hermenéutica directa de los conceptos de "revolución", "teoría" y "praxis" en Marx, MacIntyre se incorporó a la organización trotskista Socialist Labour League $(S L L)$. El escocés sintió empatía con Trotski porque representaba el desarrollo conceptual de la obra de Marx a partir de sus conceptos centrales - fundamentalmente el de "revolución" - que no permitía una estabilización ni un "aburguesamiento" partidista. En este sentido, y siempre como desarrollo hermenéutico de Marx, MacIntyre escribió una serie de artículos en la línea del $S L L$, que también se recogen en el libro.

Queda todavía mucho por estudiar sobre esta contribución original al marxismo: el concepto de revolución, o la síntesis que hizo del debate entre Cadogan, Hanson y Thompson y las líneas generales de la izquierda británica. Un último trabajo importante en este sentido fue "Breaking the Chains of Reason" (Blackledge y Davidson 2008, cap. 14), en el que - como recuerdan Blackledge y DavidsonMacIntyre recalca de nuevo su trotskismo, esta vez para contraponerlo al capitalismo liberal, representado por Keynes.

En 1961, MacIntyre dejó formalmente de ser cristiano y, al mismo tiempo, se distanció de la $S L L$ para ingresar a la Internacional Socialista. $^{2}$ A partir de esa época, como puede verse en los diferentes artículos recogidos, su marxismo se fue consumiendo, mezclado a veces con algunos comentarios periodísticos sobre la izquierda británica. MacIntyre siguió escribiendo sobre el marxismo, cada vez con menos fuerza hasta principios de los años setenta, época en la que ya se había embarcado en el proyecto que cristalizó en After Virtue.

2 (Blackledge y Davidson 2008, p. 179n). "Alasdair MacIntyre resume su propio punto de vista para nosotros: 'Era un cristiano. No. [La cuestión] es menos engañosa cuando preguntaba si era marxista para decir "sî" en lugar de "no". Pero los marxistas han sido enseñados a decir "no".'" (La traducción es mía.) 


\section{El estudio sobre el marxismo de MacIntyre}

A mi juicio, ninguno de los virajes que he comentado - dados siempre con ímpetu y ardor - se debe a un radical desacuerdo con las políticas coyunturales de cada una de estas corrientes marxistas, sino a una perenne insatisfacción de fondo, que llevó a MacIntyre a una situación límite a finales de los años sesenta, como muestra Against the Self-Images of the Age (1978). Se había consumado su ruptura con la filosofía analítica, con el marxismo, con el psicoanálisis y con el cristianismo. Creo que Blackledge y Davidson no subrayan lo suficiente esta insatisfacción, y se centran exclusivamente en la dimensión política del marxismo.

He dicho anteriormente que el libro está sesgado hacia un análisis de la obra de MacIntyre en el contexto de la izquierda inglesa, y en particular de la New Left. Si, en efecto, fuesen éstas y no MacIntyre el objeto del libro, no tendría ningún reparo en señalar las grandes virtudes en la contextualización. Sin embargo, en la introducción parece que se quiere poner énfasis en el autor y no en la época. Por esta razón me parece un análisis hasta cierto punto insuficiente, pues en la misma época en la que MacIntyre estaba - en mayor o menor medida - dedicado al marxismo, tenía otras preocupaciones intelectuales que vehiculaban y hasta llegaban a dar sentido a muchas de sus decisiones.

La compilación muestra sólo algunas de ellas, aunque Blackledge y Davidson dejan intuir otras. El libro es muy completo en el ámbito del marxismo y contiene escritos de todos los géneros: recensiones, panfletos, comentarios, artículos, capítulos de obras colectivas o fragmentos de libros de MacIntyre. Se trata de un mosaico espléndido para el lector interesado en la filosofía de este filósofo escocés o del marxismo en general. Los autores hacen un notable esfuerzo por hilvanar cada uno de esos escritos, pero el ligamen a veces parece forzado, porque no da cuenta de los otros debates que paralelamente mantuvo con los expertos en ética analítica (principalmente con Stevenson y Hare), filosofía de la religión (con Flew y Mackie) o psicoanálisis.

La temática del libro puede dividirse, a mi entender, en tres grandes áreas: la defensa del marxismo de la corriente Hegel-Marx-LeninTrotski, y la crítica del estalinismo (1953-1960), el desencanto con el marxismo y la crítica a los "intelectuales marxistas" como Marcuse, Lukács, entre otros (1960-1971) y los ensayos o comentarios críticos hacia la política del momento (1959-1973). El valor de cada uno de los escritos es, como puede imaginarse, muy desigual. Aun así, el libro - en su conjunto - tiene un gran valor, porque la selección de 
los escritos es muy adecuada, y no se han desdeñado los trabajos más breves o de corte divulgativo, si de ellos se podía extraer alguna idea interesante para caracterizar el marxismo de MacIntyre.

En este sentido, hay que destacar la acertada anotación que los compiladores han hecho de varios de los textos, cuya contextualización resulta difícil incluso para los expertos. Muchos de los escritos más breves correspondían a intervenciones radiofónicas o periodísticas que criticaban alguna cuestión referente a la actualidad política del momento. Así, resulta completamente atinada la decisión de anotar sobria pero eficazmente muchos de los escritos.

En las páginas dedicadas a la selección y a la anotación, los autores dejan entrever su innegable conocimiento de toda la obra de MacIntyre, y también recalcan que en el libro sólo aparecen aquellos escritos que tratan inequívocamente el tema del marxismo. Por ejemplo, sólo considerando la década de los cincuenta, entre sus textos se encuentran trabajos sobre metafísica, psicoanálisis, teología, ética, etc., que son altamente ilustrativos de las inquietudes de MacIntyre, cuyos vínculos con el marxismo se pierden totalmente. Sólo desde la lectura conjunta de todos esos trabajos puede el estudioso hacerse a la idea de la multiplicidad de perfiles que concurren en la obra de este filósofo escocés. ${ }^{3}$

Los lectores actuales trocean a MacIntyre a su gusto: existen muchas variantes tomistas, alguna aristotélica, y la marxista, cultivada casi exclusivamente por Blackledge y Davidson, y por alguno de sus alumnos. Falta todavía por explorar el MacIntyre de la Philosophical Theology o el psicoanalítico, por citar los más importantes. Por poner dos ejemplos: los estudios sobre el inconsciente y las pulsiones tienen un interés enorme para la ética y el marxismo, el cual cristalizó en el libro Marcuse (MacIntyre 1970); y los estudios sobre filosofía de la religión son determinantes para conocer la legitimación escatológica y su relación con el marxismo.

Lo que ocurre es que el propio MacIntyre, ya convertido al catolicismo, trazó unas líneas de estudio que han desalentado a la pléyade

\footnotetext{
${ }^{3}$ Pienso, por ejemplo, en un trabajo aparentemente tan alejado de los temas marxistas como Difficulties in Christian Belief (1959). En él se presenta un esquema analítico de los problemas clásicos de la filosofía de la religión cristiana (en debate con Flew y los filósofos de la religión de Oxford); ahí — a partir del capítulo VIIIaparecen excursos hacia el problema is-ought (central de nuevo en la filosofía moral anglosajona a partir de Wittgenstein, Hare y Stevenson) y hacia las objeciones planteadas por Marx y Freud. Esta obra permite una lectura de conjunto de las inquietudes de MacIntyre a finales de la década de los cincuenta que se encuentra en las antípodas de la descripción "política" de Blackledge y Davidson.
} 
de sus seguidores contemporáneos de hurgar en su pasado "erróneo". Y sucede que allí no había tantos "errores", ni siquiera si se mira desde su actual óptica "tomista". MacIntyre dividió su vida en tres etapas, y la primera llegaba hasta 1971, cuando se mudó a Estados Unidos. ${ }^{4}$ Blackledge y Davidson hacen una arqueología de esa etapa, contraviniendo muchas veces las directrices de MacIntyre. Es aquí donde reside otro de los mayores éxito del libro.

Por el contrario, la presentación de un MacIntyre marxista muestra sólo una de las caras de un personaje polifacético. El énfasis que ponen los autores en el compromiso político de este filósofo escocés no es un retrato preciso de su figura intelectual. Su equiparación a Thompson, a Cadogan o al propio Taylor resulta muy forzada, porque en ellos la inquietud política fue constante hasta sus últimos días, mientras que la comezón intelectual de MacIntyre era - y sigue siendo - casi exclusivamente moral.

O bien porque MacIntyre ha despreciado su obra juvenil, o bien por la dificultad de conciliar tantos problemas e inquietudes en una misma persona, el balance de estudios sobre el joven MacIntyre es bastante deficiente. Sigue faltando, hasta el día de hoy, un estudio completo sobre la obra de MacIntyre que huya de los tópicos y de las perspectivas que él mismo alienta. El mejor intento, breve y bien intencionado, fue el del malogrado profesor Émile Perreau-Saussine (2005), que procuraba dar vida a cada una de las ideas que ha tenido del escocés, con independencia de sus actuales opiniones.

Sin embargo, la gran mayoría de los textos compuestos a la vera del actual MacIntyre, por lo general muy bien escritos y muy eruditos, muestran poco interés por su primera época. Si se repasan brevemente los trabajos de síntesis de la última década, resulta muy fácil constatar que existen casi exclusivamente obras de inspiración tomista que muy pocas veces contradicen a MacIntyre.

Éste es el estado actual de los estudios sobre MacIntyre: por ejemplo, uno de los libros más brillantes sobre su filosofía moral, de Christopher Lutz (2004), apenas concede importancia al joven MacIntyre. Tampoco en el MacIntyre Reader (Knight 1998) - cuyo autor tuvo mucha influencia sobre los compiladores - se prestó demasiada atención a esta época. MacMylor (1994), en su celebrado estudio, repitió los lugares comunes del estado estandarizado de la cuestión, para no enfrentarse directamente con MacIntyre. Ni siquiera un libro tan bueno como el de Thomas D'Andrea (2006) - la mejor síntesis hasta el momento - que estudia con detalle los trabajos de juventud, se

\footnotetext{
${ }^{4}$ Véase, por ejemplo, MacIntyre 1991.
} 
separa de las directrices marcadas por la subyugante personalidad del filósofo escocés. La tesis de D'Andrea, un autor apegado a una concepción tomista, subraya que la juventud es la época de las raíces y de los fundamentos, etapa previa a la construcción de After Virtue, y que todo el proyecto intelectual anterior sólo tiene sentido a partir de los años ochenta.

Tampoco la literatura en lengua española ha sido muy pródiga en estudios sobre el joven MacIntyre. Hay que destacar, sin embargo, un libro de Javier de la Torre (2005), cuyo análisis de los años cincuenta y sesenta es bastante profundo y apunta muchos problemas que todavía permanecen sin estudiar.

El resultado es que no existe en la actualidad, por ejemplo, ningún estudio completo de las inquietudes psicoanalíticas o religiosas del joven MacIntyre. Y aún peor: hasta donde sé, tampoco hay estudios parciales sobre estas cuestiones, sino meros bocetos (Ramis Barceló 2011). No es necesario recalcar que se trata de cuestiones básicas para entender cabalmente cuáles eran los problemas de fondo sobre los que reflexionó MacIntyre y que ayudarían a comprender el pensamiento en Gran Bretaña durante los años cincuenta y sesenta.

Hace falta cierto valor para estudiar a este pensador escocés, sin caer en las redes de su arrolladora personalidad. Sus primeros escritos tienen valor per se, y no son meros fundamentos erróneos en la obra de un converso. El hecho de que MacIntyre haya concebido su vida - en estos últimos lustros - a imagen y semejanza de san Agustín no es motivo suficiente para estudiar toda su obra de juventud como un conjunto de errores de un joven descarriado. La compilación de Blackledge y Davidson — que muestra admiración en todo momento - ha sabido tratar a MacIntyre como un filósofo maduro ya en los años cincuenta y lo ha interpelado tomando como base los problemas de aquella época, y no en función de los intereses del siglo XXI.

De momento, este libro resulta una aportación fundamental, el cual se complementa con algunos artículos de los compiladores que han ido apareciendo a lo largo de esta década. De ambos se esperan aún más frutos del estudio del joven MacIntyre. Se requiere un trabajo minucioso, en el que pacientemente $-\mathrm{y}$ con libertad de criteriopuedan elaborar un estudio completo sobre temas inexplorados. Ojalá que no se detengan sólo en el marxismo y que exploren - con su método riguroso y su buen hacer- muchos de los filones que faltan para conocer mejor a Alasdair MacIntyre, uno de los grandes trashumantes de la filosofía del siglo XX. 


\section{BIBLIOGRAFÍA}

Blackledge, P., 2009, "History, Ethics and Politics", Science and Society, vol. 73, no. 1, pp. 77-84.

— 2008 , “Alasdair MacIntyre's Contribution to Marxism: A Road Not Taken", Analyse and Kritik, vol. 30, no. 1, pp. 215-227.

— 2 2005, "Freedom, Desire and Revolution: Alasdair MacIntyre's Early Marxist Ethics", History of Political Thought, vol. 26, no. 4, pp. 696720 .

Blackledge, P. y N. Davidson, 2008, Alasdair MacIntyre's Early Marxist Writings: Essays and Articles 1953-1974, Brill, Leiden.

Davidson, N., 2007, "Alasdair MacIntyre as a Marxist, 1956-1968", en K. Flett (comp.), 1956 and All That, Cambridge Scholars Publishing, Newcastle, pp. 143-165.

D'Andrea, T., 2006, Tradition, Rationality and Virtue: The Thought of Alasdair MacIntyre, Ashgate, Aldershot.

Knight, K. (comp.), 1998, The MacIntyre Reader, Polity, Cambridge.

Lutz, C., 2004, Tradition in the Ethics of Alasdair MacIntyre: Relativism, Thomism, and Philosophy, Rowman and Littlefield, Lanham.

MacIntyre, A., 1991, "An Interview with Alasdair MacIntyre", Cogito, vol. 5, no. 2, verano, pp. 67-73.

- 1978, Against the Self-Images of the Age, University of Notre Dame Press, Indiana.

— 1970, Herbert Marcuse: An Exposition and a Polemic, The Viking Press, Nueva York.

— 1959, Difficulties in Christian Belief, SCM Press, Londres.

—_, 1958, The Unconscius: A Conceptual Analysis, Routledge, Londres/NuevaYork.

- 1953, Marxism: An Interpretation, SCM Press, Londres.

McMylor, P., 1994, Alasdair MacIntyre: Critic of Modernity, Routledge, Londres.

Perreau-Saussine, E., 2005, Alasdair MacIntyre: une biographie intellectuele, Presses Universitaires de France, París.

Ramis Barceló, R., 2011, "Filosofía de la religión y mal en el joven MacIntyre", Antonianum, vol. 86 (en prensa).

— 2010, "Alasdair MacIntyre, joven lector de Freud", Isegoría: Revista de Filosofía Moral y Política, no. 42, enero-junio, pp. 231245.

Torre Díaz, J. de la, 2005, Alasdair MacIntyre, ¿Un crítico del liberalismo?, Dykinson, Madrid.

Recibido el 28 de agosto de 2009; revisado el 11 de julio de 2010; aceptado el 8 de agosto de 2010. 